\title{
Acupuncture for Chronic Low Back Pain: Recommendations to Medicare/Medicaid from the Society for Acupuncture Research
}

\author{
Jun J. Mao, MD, MSCE, Robert T. Davis, MS, LAc, ${ }^{2}$ Remy Coeytaux, MD, PhD, ${ }^{3}$ \\ Lee Hullender-Rubin, DAOM, LAc, FABORM, ${ }^{4}$ Jiang-Ti Kong, MD, \\ Hugh MacPherson, PhD, LAc, ${ }^{6}$ Vitaly Napadow, PhD, LAc, ${ }^{7}$ Rosa Schnyer, DAOM, LAc, ${ }^{8}$ \\ Peter M. Wayne, $\mathrm{PhD},{ }^{9}$ Claudia Witt, $\mathrm{MD}, \mathrm{MBA}^{10}$ and Richard Harris, $\mathrm{PhD}^{11}$
}

\begin{abstract}
Editor's Note: This column takes our series from the Society for Acupuncture Research (SAR) in a new direction. Recently, the U.S. government's Center for Medicare and Medicaid Services (CMS) issued a call for public comment related to the government's exploration of inclusion of acupuncture and licensed acupuncturists in federal payment schemes. The decision of SAR's research leaders to engage with policymakers is forward thinking, especially as much dialogue and many research needs related to acupuncture and other integrative health practices and practitioners are shifting toward implementation concerns. SAR's engagement in this arena is also reflected in the organization's 2019 conference June 27-29, 2019 in Burlington, Vermont: “Acupuncture Research, Health Care Policy, \& Community Health...Closing the Loop." The author team includes the entire SAR Board, led by president-elect Jun J. Mao, MD, and current copresidents Robert T. Davis, MS, LAc, and Richard Harris, PhD. This is also a moment to congratulate Harris on his selection to the U.S. National Institutes of Health National Advisory Council on Complementary and Integrative Health.—John Weeks, Editor-in-Chief
\end{abstract}

\footnotetext{
${ }^{1}$ Bendheim Integrative Medicine Center, Memorial Sloan Kettering Cancer Center, New York, NY.

${ }^{2}$ Acupuncture Vermont, Burlington, VT.

${ }^{3}$ Community and Family Medicine, Duke University School of Medicine, Durham, NC.

${ }^{4}$ Osher Center for Integrative Medicine, University of California San Francisco, San Francisco, CA.

${ }^{5}$ Department of Anesthesiology Pain and Perioperative Medicine, Stanford University Hospital and Clinics, Standford, CA.

${ }^{6}$ Department of Health Sciences, University of York, York, United Kingdom.

${ }^{7}$ Martinos Center for Biomedical Imaging, Massachusetts General Hospital, Boston, MA.

${ }^{8}$ School of Nursing, University of Texas, Austin, TX.

${ }^{9}$ Osher Center for Integrative Medicine, Brigham and Women's Hospital and Harvard Medical School, Boston, MA.

${ }^{10}$ Institute for Complementary and Integrative Medicine, University Hospital Zurich and University Zurich, Zurich, Switzerland.

${ }^{11}$ Departments of Anesthesiology and Internal Medicine Division of Rheumatology, University of Michigan, Ann Arbor, MI.
} 
$\mathbf{E}$ STABLISHED IN 1993, THE Society for Acupuncture Research (SAR) is a premier international research organization that is dedicated to rigorous scientific investigation and evidence-based practice of acupuncture. Its membership spans 28 countries and includes basic and translational scientists, clinical epidemiologists and trialists, and practicing acupuncturists who engage in research and education. The leadership of SAR has extensive acupuncture research expertise and has published articles in leading medical journals, such as the New England Journal of Medicine, Lancet, JAMA, Annals of Internal Medicine, as well as flagship journals for specific medical conditions such as Pain, Arthritis and Rheumatology, and Brain. We appreciate Medicare's evaluation of evidence of acupuncture for chronic low back pain in older adults. We have provided comments as follows:

A. Biological mechanisms of acupuncture: Derived from Traditional Chinese Medicine (TCM) with $>2500$ years of empirical use, acupuncture has a clear physiological basis for its therapeutic effects. In animal models, acupuncture needle stimulation stimulates endogenous opioid release into the cerebral spinal fluid that can be blocked by naloxone. ${ }^{1,2}$ In humans, brain imaging research has also found that acupuncture treatment increases opioid receptor binding in chronic pain patients with fibromyalgia. ${ }^{3}$ Translational research using functional magnetic resonance imaging in carpel tunnel patients has also found that acupuncture stimulation "quiets down" the activity of the somatosensory cortex area of the brain that seems to be hyperactive in these patients. ${ }^{4,5}$ Acupuncture also deactivates limbic brain areas, ${ }^{6}$ important for emotion and internal homeostasis, and processes that are important in chronic pain. Lastly, research in both animals and humans has also shown that acupuncture needling can modulate proteins and fibroblast cells in connective tissue, ${ }^{7,8}$ which seems to restore tissue integrity, an emerging field that is very relevant for some chronic pain conditions.

B. Clinical evidence of acupuncture: Following are some key studies demonstrating that acupuncture is safe, effective, and cost-effective for chronic pain. Although many of these studies included older adults, very little research has focused exclusively on people aged 65 years or older.

1. Acupuncture is safe: In a cumulative review of more than 1 million acupuncture treatments, the risk of a serious adverse event with acupuncture was estimated to be 0.05 per 10,000 treatments and 0.55 per 10,000 individual patients. Most common side effects were bleeding at needle site and localized needling pain. ${ }^{9}$ Similar findings were also reported in 229,230 patients with more than 2 million visits. ${ }^{10}$

2. Acupuncture is effective for chronic pain, including low back pain ${ }^{11}$ : In patient-level meta-analyses of randomized controlled trials that included $\sim 18,000-21,000$ patients with chronic musculoskeletal pain, acupuncture was found to be substantially better than standard care and significantly better than sham acupuncture. ${ }^{12,13}$ Approximately $20 \%$ of the population included in these trials are aged 65 years or older.

3. The beneficial effects of acupuncture persist beyond the course of treatment: In a meta-analysis of 17,922 patients with chronic nonmalignant pain, $~ 90 \%$ of the pain-relieving effects of acupuncture relative to controls were sustained at 12 months. ${ }^{14}$

4. Acupuncture is cost-effective for chronic pain management: In two randomized trials that included $>12,000$ patients with low back pain, acupuncture treatment was associated with clinically meaningful improvement in quality of life with a modest incremental increase in cost greatly below the $\$ 50,000$ cost per quality ratio. ${ }^{15,16}$

C. Recommendations for inclusion of education requirements for acupuncture providers: There are $\sim 37,000$ licensed acupuncturists (LAcs) in the United States, who received comprehensive clinical $(660 \mathrm{~h})$ and didactic $(1245 \mathrm{~h})$ acupuncture training and have an established master's level accredited education and certification process. LAcs are recognized as qualified providers of acupuncture by the Department of Veteran Affairs, 47 states and the District of Columbia, as well as many thirdparty payers throughout the country. Physician-acupuncturists typically complete a 200 - or $300-\mathrm{h}$ continuing medical education training program focused on acupuncture. To meet the need of care delivery to Medicare patients, we propose the inclusion of both LAcs and physician acupuncturists as a Centers for Medicare and Medicaid Services (CMS)-covered service provider for acupuncture. SAR also recommends additional training for all acupuncturists in the biological mechanisms and clinical evidence of acupuncture for pain management, as well as optimal interprofessional and patient communication to enhance the delivery of safe effective acupuncture treatments.

\section{Recommendations for Medicare's evaluation of acupuncture for chronic low back pain:}

1. SAR recommends pragmatic comparative effectiveness trials of acupuncture against standard medical care (drugs) or active therapeutic interventions such as physical therapy and cognitive behavioral therapy that have a well-established evidence base.

2. SAR recommends studies to capture patient-centered and health system-relevant outcomes in addition to pain, such as polypharmacy, blood pressure, and falls.

3. SAR recommends thoughtful incorporation of validated expectancy and preference measures to understand how these may affect trial results as well as guide patient-centered care.

In summary, SAR supports Medicare's evaluation of evidence of acupuncture for chronic low back pain. With population expansion and aging, 78 million Americans will be 65 years or older by 2035 . Opioid therapy has limited efficacy for chronic musculoskeletal pain and can increase risk of oversedation and falls that threaten the functional independence of older persons. Nonsteroidal anti-inflammatory drugs have gastrointestinal and renal side effects, and increase cardiovascular morbidity and mortality, the leading cause of death in the United States. Its biological mechanism, combined with growing clinical evidence, makes acupuncture a potential treatment to be considered as part of comprehensive pain management 
strategy for millions of older Americans. This option will ensure that older Americans have safe and effective treatments to manage chronic low back pain and improve their function and quality of life. SAR is willing to be engaged during this review process to provide our expertise and perspective.

\section{References}

1. Cheng RS, Pomeranz B. Electroacupuncture analgesia could be mediated by at least two pain-relieving mechanisms; endorphin and non-endorphin systems. Life Sci 1979;25:1957-1962.

2. Han JS. Acupuncture and endorphins. Neurosci Lett 2004; 361:258-261.

3. Harris RE, Zubieta JK, Scott DJ, et al. Traditional Chinese acupuncture and placebo (sham) acupuncture are differentiated by their effects on mu-opioid receptors (MORs). Neuroimage 2009;47:1077-1085.

4. Napadow V, Liu J, Li M, et al. Somatosensory cortical plasticity in carpal tunnel syndrome treated by acupuncture. Hum Brain Mapp 2007;28:159-171.

5. Maeda Y, Kim H, Kettner N, et al. Rewiring the primary somatosensory cortex in carpal tunnel syndrome with acupuncture. Brain 2017;140:914-927.

6. Hui KK, Liu J, Makris N, et al. Acupuncture modulates the limbic system and subcortical gray structures of the human brain: Evidence from fMRI studies in normal subjects. Hum Brain Mapp 2000;9:13-25.

7. Langevin HM, Churchill DL, Cipolla MJ. Mechanical signaling through connective tissue: A mechanism for the therapeutic effect of acupuncture. FASEB J 2001;15:22752282.

8. Langevin HM, Churchill DL, Wu J, et al. Evidence of connective tissue involvement in acupuncture. FASEB J 2002;16:872-874.
9. White A. A cumulative review of the range and incidence of significant adverse events associated with acupuncture. Acupuncture Med 2004;22:122-133.

10. Witt CM, Pach D, Brinkhaus B, et al. Safety of acupuncture: Results of a prospective observational study with 229,230 patients and introduction of a medical information and consent form. Forsch Komplementarmed 2009;16:91-97.

11. Berman BM, Langevin HM, Witt CM, Dubner R. Acupuncture for chronic low back pain. N Engl J Med 2010; 363:454-461.

12. Vickers AJ, Cronin AM, Maschino AC, et al. Acupuncture for chronic pain: Individual patient data meta-analysis. Arch Intern Med 2012;172:1444-1453.

13. Vickers AJ, Vertosick EA, Lewith G, et al. Acupuncture for chronic pain: Update of an individual patient data metaanalysis. J Pain 2018;19:455-474.

14. MacPherson H, Vertosick EA, Foster NE, et al The persistence of the effects of acupuncture after a course of treatment: A meta-analysis of patients with chronic pain. Pain 2017;158:784-793.

15. Ratcliffe J, Thomas KJ, MacPherson H, Brazier J. A randomised controlled trial of acupuncture care for persistent low back pain: Cost effectiveness analysis. BMJ 2006;333: 626.

16. Witt CM, Jena S, Selim D, et al. Pragmatic randomized trial evaluating the clinical and economic effectiveness of acupuncture for chronic low back pain. Am J Epidemiol 2006; 64:487-496.

Address correspondence to: Jun J. Mao, MD, MSCE Bendheim Integrative Medicine Center 1429 First Avenue New York, NY 10021

E-mail:maoj@mskcc.org 